\title{
Líquen plano: caso-clínico sobre a perspetiva dos cuidados de saúde primários
}

Catarina Ornelas, ${ }^{1}$ Ana Rodrigues ${ }^{2}$

\section{RESUMO}

Introdução: O líquen plano (LP) é uma doença inflamatória crónica que pode envolver a pele e mucosas oral, esofágica, laríngea, genital e conjuntivas e cuja prevalência é estimada entre 0,22-5\% da população mundial.

Descrição do caso: Homem de 32 anos, sem antecedentes pessoais relevantes, recorreu à consulta da Unidade de Saúde Familiar (USF) por lesões cutâneas pruriginosas que mantinha desde há três semanas, de início nos tornozelos e com disseminação posterior para os punhos, antebraços e tronco, bilateralmente. Negava contacto com fármacos, relações sexuais de risco e outros sintomas. Automedicou-se com benzoato de benzilo durante três dias e, por ausência de resolução das queixas, recorreu ao serviço de urgência geral, tendo sido diagnosticado com eczema de contacto e medicado com mometasona creme, também sem resposta. À observação apresentava pápulas eritematovioláceas generalizadas, simétricas, com fenómeno de Koebner, sendo a maior incidência nos punhos e poupando o couro cabeludo, as palmas das mãos e as plantas dos pés. Nos tornozelos apresentava ainda placas eritematosas e descamativas em zona de pele xerótica. Apresentava uma lesão na glande de cerca de $1 \mathrm{~cm}$, com limite bem definido, ligeiramente descamativo, com centro de aspeto cicatricial. Foram colocadas como hipóteses diagnósticas: sífilis secundária, pitiríase rósea e LP. Pelas características clínicas, a pitiríase rósea foi prontamente excluída. Foram pedidas serologias para infeções sexualmente transmissíveis e medicado com hidroxizina e creme hidratante. As serologias foram negativas, obviando o diagnóstico de LP. O utente foi referenciado à dermatologia para instituição de terapêutica sistémica.

Comentário: O presente caso clínico é um reflexo da importância de uma anamnese e exame objetivos completos e de um alto índice de suspeição naquela que é uma patologia pouco comum nos cuidados de saúde primários. Também realça a importância do médico de família como gestor dos cuidados de saúde dos utentes.

Palavras-chave: Líquen plano; Diagnóstico; Terapêutica

\section{INTRODUÇÃO}

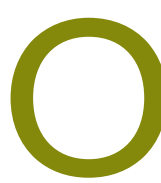

líquen plano (LP) é uma doença inflamatória crónica que pode envolver pele e mucosas e cuja prevalência é estimada entre $0,22-5 \%$ da população mundial. ${ }^{1-3}$

É caracteristicamente simétrica e surge na superfície flexora dos membros, podendo ainda acometer as unhas, o couro cabeludo, as mucosas oral, esofágica, laríngea, genital e conjuntivas. ${ }^{1-3}$ É conhecida como a doença dos seis $\mathrm{P}$ - placas e pápulas, purpúricas, poligonais, planas, pruriginosas -, que podem apresentar linhas estriadas conhecidas por estrias de Wickham. ${ }^{2}$

1. Médica Interna de Medicina Geral e Familiar. USF Magnólia, ACeS Loures-Odivelas 2. Médica Assistente Graduada. USF Magnólia, ACeS Loures-Odivelas.
A medicina geral e familiar (MGF) é a porta de entrada no Sistema Nacional de Saúde e é o médico de família que lida com todas as doenças em todas as suas fases de evolução, sendo muitas vezes aquele que se depara com as suas primeiras manifestações.

A exposição às patologias menos frequentes torna a suspeita clínica a principal aliada no diagnóstico.

Serve o presente relato de caso para expor a história de um caso de LP, onde o alto índice de suspeição foi a chave do diagnóstico.

\section{DESCRIÇÃO DO CASO}

Homem de 32 anos, caucasiano, residente em Sintra e que se insere numa família nuclear, funcional, na fase I do Ciclo Familiar de Duvall e pertencente à classe II 
de Graffar, correspondente à classe média-alta. Sem antecedentes pessoais relevantes e sem hábitos etanólicos, tabágicos ou consumo de drogas. Dos antecedentes familiares destaca-se a mãe com asma, rinite alérgica, hipertensão arterial e dislipidemia e o pai com rinite alérgica, asma, dislipidemia, osteoartrose e excesso de peso.

O utente é utilizador da USF habitualmente para situações pontuais de caráter agudo. Em 22/11/2017 recorreu a uma consulta programada na unidade, apresentando queixas de lesões cutâneas pruriginosas que mantinha desde há três semanas. As lesões tiveram início nos tornozelos e depois disseminaram para os punhos, antebraços e tronco, bilateralmente. Mencionava ainda uma lesão no pénis, descamativa e indolor. Por iniciativa própria automedicou-se com benzoato de benzilo durante três dias, sem melhoria clínica, tendo posteriormente recorrido a uma consulta de urgência geral que colocou eczema de contacto como hipótese diagnóstica, prescrevendo mometasona creme e bilastina $20 \mathrm{mg}$, que cumpriu, sem resposta ao tratamento.

Negava terapêutica habitual, contexto epidemiológico e infeções virais recentes. Negava febre, astenia, náuseas, vómitos, diarreia, dispneia, tosse, exsudado uretral ou outros. Negava ainda contactos sexuais de risco.

Ao exame objetivo apresentava pápulas eritematovioláceas generalizadas, simétricas, algumas dispostas de forma linear, sugerindo fenómeno de Koebner, sendo a maior incidência nos punhos (Figura 1) e poupando o couro cabeludo, palmas das mãos e plantas dos pés. Nos tornozelos apresentava ainda placas eritematosas e descamativas em zona de pele xerótica (Figura 2). A lesão da glande media cerca de $1 \mathrm{~cm}$ de maior diâmetro, com limite bem definido, esbranquiçado, ligeiramente descamativo, com centro de aspeto cicatricial. A cavidade oral e as unhas não apresentavam alterações.

Ao formular as hipóteses diagnósticas foram imediatamente consideradas aquelas que são as patologias dermatológicas de acometimento generalizado da epiderme, nomeadamente: urticária, toxidermia, escabiose, sífilis secundária, pitiríase rósea, psoríase e foi ainda colocada a hipótese de LP. Pela história clínica discordante e características das lesões foram excluídas a urticária, toxidermia, escabiose, psoríase e pitiríase

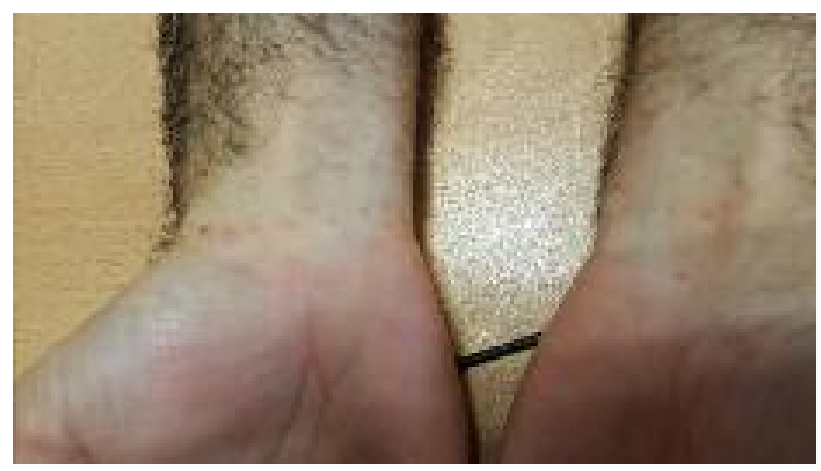

Figura 1.22 de novembro; Pápulas eritematovioláceas na face interna dos punhos previamente à instituição de $10 \mathrm{mg}$ de acitretina.

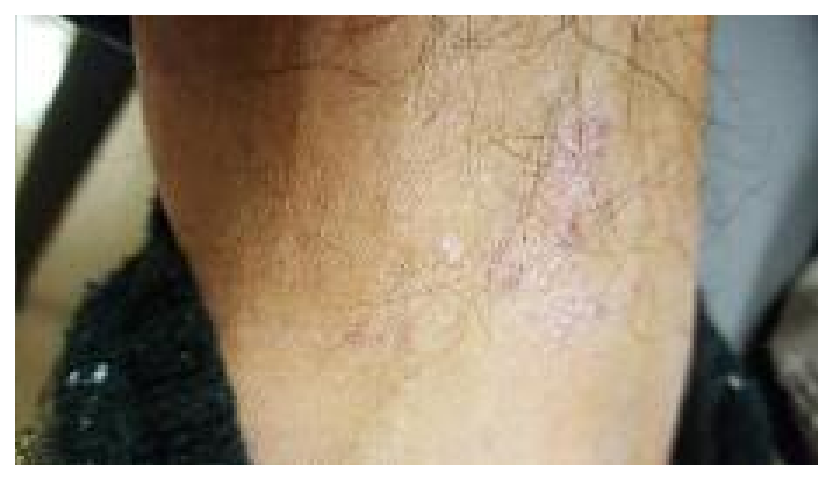

Figura 2. 22 de novembro; Pápulas confluentes em zona de xerose cutânea na face interna do tornozelo, previamente à instituição de $10 \mathrm{mg}$ de acitretina.

rósea, tornando a sífilis secundária e o LP as mais prováveis.

Nesse sentido foram solicitados exames complementares de diagnóstico, nomeadamente a avaliação analítica com VDRL, TPHA, VIH 1 e 2, Ag HBs, anti-HCV, pesquisa de DNA de clamídia na urina e pesquisa de gonorreia, permitindo, assim, a confirmação/exclusão diagnóstica de sífilis e de outras infeções sexualmente transmissíveis. Foram explicadas ao utente as hipóteses diagnósticas prováveis, tornando-se a sífilis secundária geradora de grande ansiedade e preocupação por parte do utente.

Como tratamento sintomático foi-lhe aconselhada a aplicação de creme hidratante e foi dada indicação de manutenção da bilastina $20 \mathrm{mg}$, adicionando-se a hidroxizina 25mg à noite. Remarcou-se uma consulta para duas semanas depois, para reavaliação e apresentação dos resultados analíticos. 


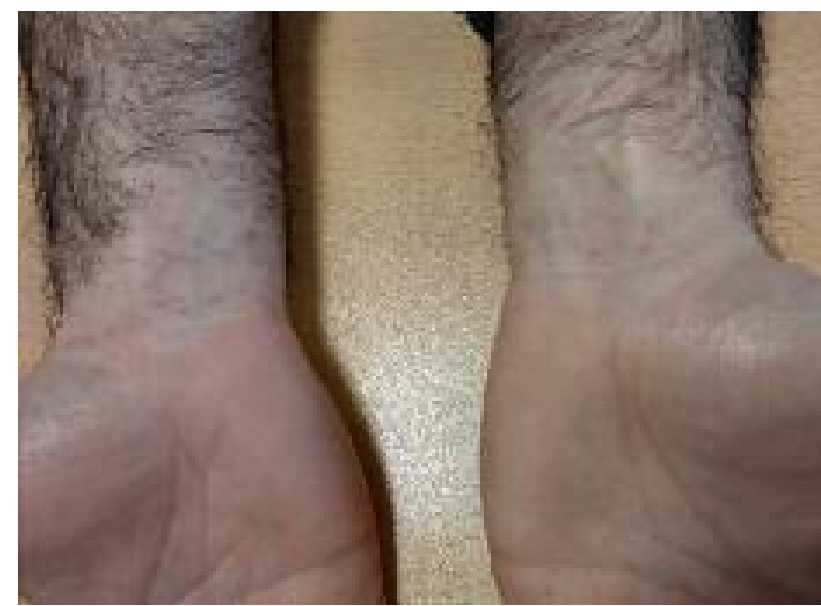

Figura 3. 11 de abril; Pápulas eritematovioláceas em involução na face interna dos punhos, após medicação com $10 \mathrm{mg}$ de acitretina.

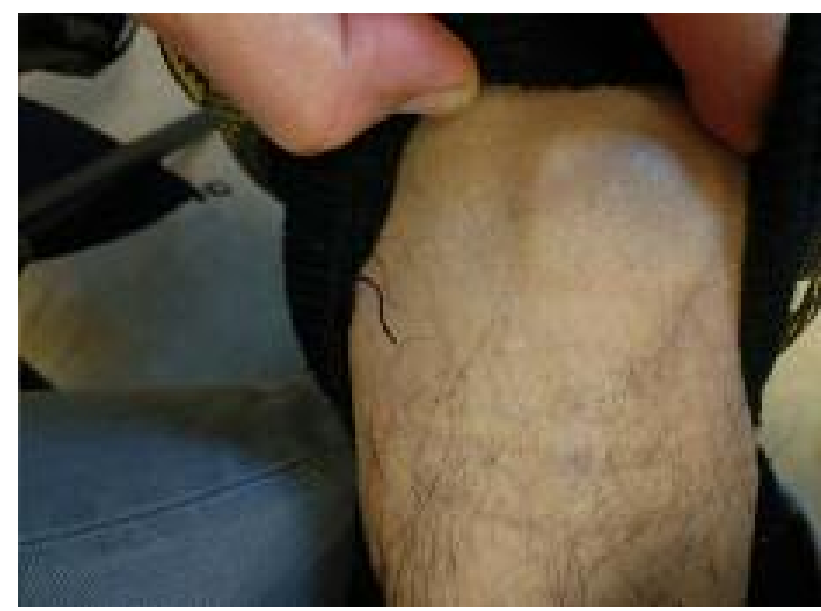

Figura 4. 11 de abril; Xerose cutânea residual na face interna do tornozelo, após medicação com 10 mg de acitretina.

Na consulta de reavaliação, o utente mantinha as queixas, com lesões cutâneas pruriginosas com as mesmas características e referiu o aparecimento de nova lesão na pálpebra superior esquerda.

À observação mantinha as pápulas eritematovioláceas identificadas em consulta prévia, com melhoria das lesões dos tornozelos, agora cicatriciais. Na glande conservava a placa descamativa e esbranquiçada prévia. A cavidade oral não apresentava alterações visíveis.

Analiticamente encontrava-se normal, sem positividade dos resultados para as infeções sexualmente transmissíveis. Posto isto, excluiu-se sífilis secundária, tornando-se o LP a hipótese diagnóstica mais provável.
Dado carácter generalizado das lesões optou-se por não aplicar corticoide tópico, ficando medicado sintomaticamente com hidroxizina $25 \mathrm{mg}$ de $12 / 12 \mathrm{~h}$ e creme hidratante. Nesta fase, um médico de família com experiência em doenças da pele poderia ter iniciado a terapêutica dirigida atempadamente, com referenciação subsequente para a dermatologia. Contudo, pelo pouco conhecimento no manuseio desta situação, a referenciação impôs-se.

O utente foi informado sobre a hipótese diagnóstica mais provável, a ser confirmada pela dermatologia, e foi explicada a necessidade e o tipo de tratamento a ser iniciado na especialidade hospitalar. Foram esclarecidas as dúvidas colocadas e verificou-se uma aceitação e tranquilização por parte do utente. Foi encaminhado à consulta de dermatologia do hospital de referência, tendo sido observado no espaço de um mês, com a confirmação clínica de LP e diagnosticado também LP oral. Foi medicado com acitretina $10 \mathrm{mg} /$ dia e betametasona creme, com reavaliação em um mês.

Na consulta de reavaliação em dermatologia mantinha xerose cutânea e algumas pápulas, mas menos pruriginosas. Teve indicação para manter o tratamento e foi agendada nova consulta.

Dois meses depois, o utente recorreu à USF preocupado por apresentar desconforto orofaríngeo à direita, que descrevia como um incómodo, com um mês de evolução e sem resposta à dose adequada de anti-inflamatório durante três dias. À observação apresentava-se sem alterações orofaríngeas, sublinguais e do palato. Apesar de se considerar a possibilidade de rinite alérgica e refluxo gastroesofágico, foi também colocada como hipótese diagnóstica a extensão orofaríngea do LP oral. Assim, foi feita prova terapêutica com inibidor da bomba de protões durante 14 dias e, em caso de não resolução, posterior prova terapêutica com anti-histamínico e referenciação à otorrinolaringologia. Nesta consulta as lesões de LP estavam francamente melhoradas, com ênfase para os punhos e tornozelos que apresentavam apenas lesões residuais (Figuras $3 \mathrm{e}$ 4). Contudo, surgiram ainda novas lesões na face e couro cabeludo.

Como plano, o utente mantém consultas de reavaliação regulares em dermatologia para vigilância clínica e dos efeitos da medicação prescrita, cujas doses poderão ser ajustadas consoante a resposta ao trata- 
mento, que deverá ser cumprido até resolução do quadro.

\section{COMENTÁRIO}

As doenças do foro dermatológico ocupam uma vasta área da prática clínica do médico de família. ${ }^{4-5}$

De facto, patologias como pitiríase rósea, sífilis secundária, LP e até mesmo toxidermias não são habitualmente encontradas nas consultas de MGF de uma forma geral; assim, a formulação destas hipóteses diagnósticas requer alto índice de suspeição, de informação, de revisão e de atualização constantes na área. Por este motivo, são preponderantes para o manuseio das doenças do foro dermatológico a realização de estágios e de cursos de dermatologia por parte dos internos de MGF e dos médicos de família.

O LP é uma doença inflamatória crónica, imunomediada, que pode afetar a pele e as mucosas, as unhas e o couro cabeludo..$^{1-3}$ Atinge mais comummente adultos de meia-idade, de ambos os géneros, mas é mais predominante em mulheres. ${ }^{2}$ Pode manifestar-se de diferentes formas, sendo a classificação orientada com base nas características das lesões ou na localização das mesmas. ${ }^{1}$ Tendo em conta a morfologia das lesões poder-se-á classificar o LP em: papular (clássico), linear, anular, atrófico, hipertrófico, folicular, pigmentoso, pigmentoso-inverso e vesicobulhoso. ${ }^{1}$

Apesar de a maioria dos casos de LP ser idiopática, é importante considerar a relação com a ingestão de certos medicamentos (antimaláricos, penicilaminas, diuréticos tiazídicos, betabloqueantes, anti-inflamatórios não esteroides, inibidores da enzima conversora da angiotensina, entre outros). ${ }^{2}$

A sua apresentação clássica é caracterizada pelos seis $\mathrm{P}$ - placas e pápulas, purpúricas, poligonais, planas, pruriginosas -, com afeção simétrica das superfícies flexoras e com presença de estrias de Wickham e do fenómeno de Koebner.$^{1-3}$ É tipicamente muito pruriginoso e de diagnóstico clínico, ${ }^{1-2}$ passível de ser realizado por um médico de família informado, devendo a biópsia ser considerada nos casos mais atípicos. ${ }^{1,7}$

Contudo, a realização de exames complementares de diagnóstico não deve ser desprezada. O estudo de infeções sexualmente transmissíveis é fundamental, não só pelo diagnóstico diferencial com a sífilis secundária, mas também pela associação, ${ }^{1-2}$ estatisticamente signi- ficativa, descrita entre a presença do vírus da hepatite C e o desenvolvimento de LP. . $-3,7-8^{7}$

Apesar de muitas vezes ter resolução espontânea num período entre seis meses a um ano, há tendência para a recorrência, sobretudo nos casos de LP generalizado. ${ }^{1}$

Uma vez que os dados de ensaios clínicos aleatorizados controlados são escassos, o tratamento é baseado na experiência clínica. ${ }^{9}$ Os corticoides tópicos de potência elevada, como o clobetasol, ${ }^{2}$ aplicados uma a duas vezes por dia ao longo de duas a três semanas, constituem a primeira linha do tratamento do LP da pele localizado e da mucosa genital, ${ }^{2,7,9}$ situação que pode ser orientada nos cuidados de saúde primários. $\mathrm{O}$ controlo do prurido pode ser feito com recurso a anti-histamínicos como a hidroxizina. ${ }^{7}$

A referenciação à dermatologia impõe-se quando não há resposta à terapêutica tópica ou quando a extensão da doença implica um tratamento sistémico (como a corticoterapia oral ou a acitretina, por exemplo). ${ }^{2,7,9} \mathrm{~A}$ corticoterapia oral corresponde a uma dose de $0,5-1 \mathrm{mg} / \mathrm{kg}$ a realizar diariamente durante quatro a seis semanas. ${ }^{9}$ Por sua vez, a dose de acitretina que mostrou benefício em relação ao placebo num ensaio aleatorizado controlado foi a de $30 \mathrm{mg}$ por dia durante oito semanas. ${ }^{9}$ No presente caso clínico, a dose diária utilizada foi a de $10 \mathrm{mg}$, a ajustar posteriormente consoante a resposta clínica.

Em suma, a descrição do presente caso serviu para a revisão da abordagem diagnóstica e terapêutica do LP. Destaca-se pelo papel crucial do médico de família no diagnóstico precoce desta entidade e pela articulação rápida e eficaz com os cuidados de saúde secundários, garantindo o bem-estar do doente.

Evidencia ainda o papel da MGF como gestora dos problemas de saúde dos seus utentes, podendo interligar as várias áreas médicas na elaboração de hipóteses diagnósticas.

Ao médico de família cabe não só o estabelecimento de diagnósticos e sua orientação terapêutica, mas ainda o aconselhamento e advocacia nos cuidados de saúde prestados, tendo sido, neste caso, crucial para o esclarecimento das dúvidas do utente e na gestão da ansiedade face ao problema diagnosticado.

Por fim, o conhecimento sobre as dermatoses mais frequentes nos cuidados de saúde primários é impor- 
tante para dirigir a formação da MGF e tornar os médicos de família mais capazes no seu diagnóstico e abordagem.

\section{REFERÊNCIAS BIBLIOGRÁFICAS}

1. Gorouhi F, Davari P, Fazel N. Cutaneous and mucosal lichen planus: a comprehensive review of clinical subtypes, risk factors, diagnosis, and prognosis. Sci World J. 2014;2014:ID742826.

2. Katta R. Lichen planus. Am Fam Physician. 2000;61(11):3319-28.

3. Gupta S, Jawanda MK. Oral lichen planus: an update on etiology, pathogenesis, clinical presentation, diagnosis and management. Indian J Dermatol. 2015;60(3):222-9.

4. Awadalla F, Rosenbaum DA, Camacho F, Fleischer JrAB, Feldman SR. Dermatologic disease in family medicine. Fam Med. 2008;40(7):507-11.

5. Rübsam ML, Esch M, Baum E, Bösner S. Diagnosing skin disease in primary care: a qualitative study of GPs' approaches. Fam Pract. 2015; 32(5):591-5.

6. Ortiz A, Herrera T, Pérez del Molino C, Piñeiro F, Perales ML, Muñoz P. Epidemiología de las enfermedades dermatológicas en atención pri- maria [Epidemiology of skin diseases in primary health care]. Rev San Hig Pub. 1992;66(1):71-82. Spanish

7. Usatine R, Tinitigan M. Diagnosis and treatment of lichen planus. Am Fam Physician. 2011;84(1):53-60.

8. Shengyuan L, Songpo Y, Wen W, Wenjing T, Haitao Z, Binyou W. Hepatitis $C$ virus and lichen planus: a reciprocal association determined by a meta-analysis. Arch Dermatol. 2009;145(9):1040-7.

9. Le Cleach L, Chosidow O. Clinical practice: lichen planus. N Engl J Med. 2012;366(8):723-32.

\section{CONFLITO DE INTERESSES}

Os autores declaram não ter quaisquer conflitos de interesse.

\section{ENDEREÇO PARA CORRESPONDÊNCIA}

Catarina Ornelas

E-mail: catarina.orn@gmail.com

https://orcid.org/0000-0001-6726-3567

Recebido em 29-05-2018

Aceite para publicação em 02-07-2019

\section{ABSTRACT}

\section{LICHEN PLANUS: A PRIMARY CARE PERSPECTIVE CASE REPORT}

Introduction: Lichen planus (LP) is an inflammatory chronic disorder that can involve the skin, esophageal, laryngeal, genital and the conjunctival mucous membranes. The estimated prevalence is in the range of 0.22 to $5 \%$ worldwide.

Case description: Thirty-two years-old man with no relevant personal background, showed up to a primary care medical appointment complaining about pruritic cutaneous lesions with 3-weeks duration. They began in the ankles and spread to the wrists, forearms, and trunk, bilaterally. He denied contact with drugs, risky sexual exposure and other symptoms. The patient started by himself a 3-day course of benzyl benzoate. Since symptoms remained, he pursued the emergency department for help. He was diagnosed with contact eczema and medicated with mometasone cream without response. The physical exam revealed a symmetrical erythematous papule exanthem with the Koebner phenomenon, being the wrists the most problematic areas. It didn't affect the scalp, plants nor the palms. The ankle showed erythematous scaly plaques and xerotic skin. He also presented a glans penis lesion measuring about $1 \mathrm{~cm}$, with well-defined limits, slightly desquamative, with a cicatricial aspect of the centre. The following diagnostic hypotheses were assumed: secondary syphilis, pityriasis rosea, and LP. Due to clinical characteristics, pityriasis rosea was promptly excluded. Sexually transmitted diseases exams were performed and the patient medicated with hydroxyzine and moisturizing cream. Exams came negative, becoming clear an LP diagnosis. The patient was referenced to Dermatology to start systemic therapy.

Commentary: The present clinical report reminds the relevance of a complete physical exam and anamnesis with a high suspicion index in the diagnosis of rare conditions in primary care. Also, it enhances the family physician's importance in managing patient health care.

Keywords: Lichen planus; Diagnostic; Therapeutics 